\title{
Efficient high-throughput sequencing of a laser microdissected chromosome arm
}

\author{
Eva Seifertova ${ }^{1 *}$, Lyle B Zimmerman ${ }^{2}$, Michael J Gilchrist ${ }^{2}$, Jaroslav Macha', Svatava Kubickova ${ }^{3}$, \\ Halina Cernohorska ${ }^{3}$, Vojtech Zarsky ${ }^{1}$, Nick DL Owens², Abdul K Sesay², Tereza Tlapakova ${ }^{1}$ and Vladimir Krylov ${ }^{1}$
}

\begin{abstract}
Background: Genomic sequence assemblies are key tools for a broad range of gene function and evolutionary studies. The diploid amphibian Xenopus tropicalis plays a pivotal role in these fields due to its combination of experimental flexibility, diploid genome, and early-branching tetrapod taxonomic position, having diverged from the amniote lineage 360 million years ago. A genome assembly and a genetic linkage map have recently been made available. Unfortunately, large gaps in the linkage map attenuate long-range integrity of the genome assembly.

Results: We laser dissected the short arm of $X$. tropicalis chromosome 7 for next generation sequencing and computational mapping to the reference genome. This arm is of particular interest as it encodes the sex determination locus, but its genetic map contains large gaps which undermine available genome assemblies. Whole genome amplification of 15 laser-microdissected $7 p$ arms followed by next generation sequencing yielded $\sim 35$ million reads, over four million of which uniquely mapped to the $X$. tropicalis genome. Our analysis placed more than 200 previously unmapped scaffolds on the analyzed chromosome arm, providing valuable low-resolution physical map information for de novo genome assembly.
\end{abstract}

Conclusion: We present a new approach for improving and validating genetic maps and sequence assemblies. Whole genome amplification of 15 microdissected chromosome arms provided sufficient high-quality material for localizing previously unmapped scaffolds and genes as well as recognizing mislocalized scaffolds.

Keywords: Xenopus, Tropicalis, Chromosomes, Next generation sequencing, WGA, Genetic map

\section{Background}

Recently, complete genomes of many important model organisms have been assembled using either Sanger or next generation sequencing such as Solexa (Illumina), Roche 454, SOLiD etc. [1]. However, repetitive elements in higher eukaryotic genomes interfere with assembly of sequence information alone into unified chromosomescale scaffolds [2]. This obstacle is usually overcome by construction of physical or meiotic linkage maps to provide long-range contiguity. Physical mapping can be accomplished by a variety of methods including restriction analysis of BAC libraries, radiation hybrid panels, and direct visualization of marker positions on chromosomes using fluorescent in situ hybridization (FISH). The latter

\footnotetext{
* Correspondence: eva.seifertova@natur.cuni.cz

${ }^{1}$ Charles University in Prague, Faculty of Science, Vinicna 7, Prague 2128 44, Czech Republic

Full list of author information is available at the end of the article
}

approach is quite accurate, but only a few markers can be localized in one run. In meiotic linkage mapping, relationships among polymorphic marker sequences are determined by relative frequency of recombination. However, recombination frequency is highly variable, often decreasing near centromeres and high in hotspots, making it difficult to compare genetic and physical distances. In addition, resolution of linkage analysis depends on the type of markers chosen and their abundance.

The diploid amphibian Хеnopus tropicalis plays a key role in basic biological research. This model system is particularly valuable for studies of early vertebrate embryonic development [3,4], functional genomics [5,6], cell biology [3,7], and vertebrate genome evolution [8]. Its $1.7 \times 10^{9}$ bp genome was sequenced [9] and a genetic map covering its 10 chromosomes was constructed [10]. Two genome assemblies are in wide use, both available on www.xenbase.org. The version 4.1 assembly (v4.1,

\section{Biomed Central}


Joint Genome Institute) is solely sequence-based and consists of 19,501 scaffolds. A more recent assembly, version 7.1 (v7.1, [9], discussed in [11]), orders reassembled scaffolds using meiotic map and synteny information into a 'main assembly' of 10 chromosome-scale superscaffolds covering $\sim 75 \%$ of the genome, with another $\sim 7000$ small 'orphan' scaffolds not incorporated into the main assembly. While this long-range assembly is extremely useful, regions assembled by inferring shared gene order with more complete amniote assemblies must be considered provisional, as synteny is not always conserved over large phylogenetic distances. Likewise, the genetic map only locates v4.1 scaffolds covering $\sim 62 \%$ of the $X$. tropicalis genome, or about 758 of $1300 \mathrm{v} 4.1$ [10] scaffolds larger than $100 \mathrm{~kb}$; polymorphic markers were not obtained for the remaining 'unmapped scaffolds'. The largest gaps in the genetic map include the entire short arm of chromosome 2 , and a $\sim 15 \mathrm{cM}$ span inside the distalmost marker on the $\mathrm{p}$ arm of chromosome 7. Interestingly, the gap on chromosome 7 appears to contain the $X$. tropicalis sex determining locus [12], although an independent marker analysis suggests that there is not a large region of sexspecific sequence [13] which might interfere with meiotic mapping.
To identify sequences within these gaps as well as map and assembly errors, we developed an improved method based on high-throughput sequencing of laser microdissected chromosome arms (Figure 1). Recent technical advances have enabled low cost genome sequencing of nearly any species $[14,15]$. However, direct sequencing of specific chromosomes or chromosomal regions has only been successful in species where individual chromosomes could be separated by flow sorting (reviewed in [16]). Microdissection of chromosomes has been attempted, but this approach depends on whole-genome amplification due to practical limits on the amount of starting material [17]. In the only published study, sequence resolution was low, probably due to the poor yield and quality of the DNA obtained [18].

Here we used 15 microdissected copies of the short arm of chromosome 7. This small amount of material was then subjected to whole genome amplification (WGA), and sequencing libraries were constructed by transposase-based simultaneous fragmentation and primer insertion, and then sequenced. For WGA, we chose the Sigma GenomePlex single cell kit since it amplified more markers and yielded the highest quantity of DNA relative to other systems [19], and has been successfully

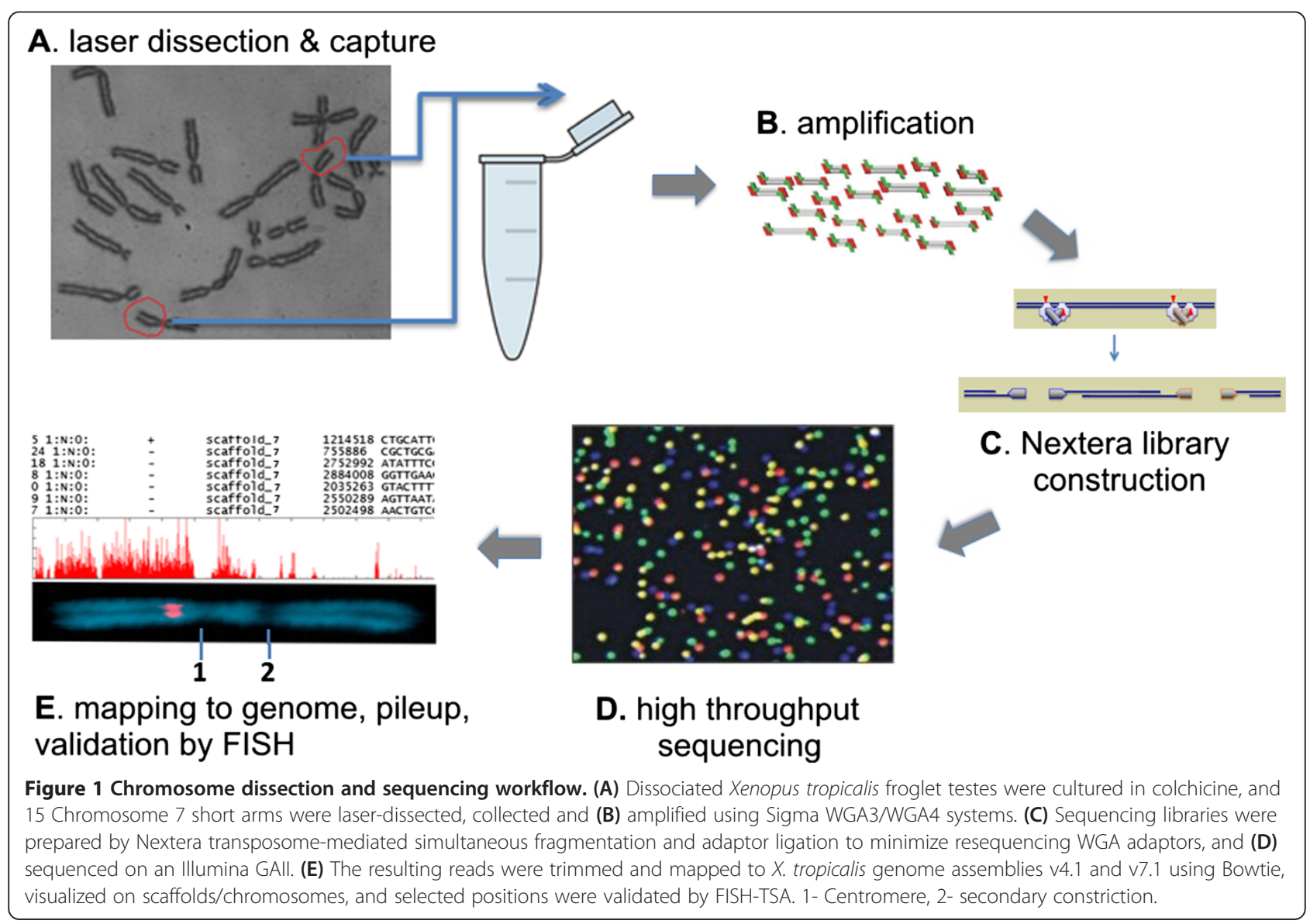


used to amplify microdissected human chromosomes for SNP genotyping [17]. This combination provided excellent read depth for placing previously unmapped scaffolds and genes to the $7 \mathrm{p}$ region, as well as recognizing mislocalized scaffolds.

\section{Results}

We prepared metaphase chromosomes from primary cell cultures of dissected subadult frog testes [19]. We then laser microdissected 15 copies of the short arm of chromosome 7, which is easily distinguishable due to a secondary constriction in its q arm (Figure 1E). To ensure harvest of the entire $\mathrm{p}$ arm, the laser path was targeted between the constriction and centromere (Figure 1E). The extremely small amount of starting material was then amplified by WGA, with a total yield of $\sim 20 \mu \mathrm{g}$ averaging $500-600$ bp in size. For library construction, we wished to minimize resequencing WGA primers added to ends of genomic fragments. To that end, we used a transposasebased simultaneous fragmentation/adaptor ligation method (Nextera, Illumina Inc.) where sequencing primer insertions are biased away from DNA ends. 80 bp reads were then obtained in a single lane of Illumina GAII. Reads were mapped to both versions of Xenopus tropicalis assemblies (v4.1 and v7.1) using Bowtie.

\section{Comparison to v4.1 assembly}

In total, we obtained 35 million 80 bp reads, $18 \%$ of which mapped with a maximum of $2 / 80$ mismatches to at least one location in the $v 4.1$ genome assembly. Of these, $3,900,340$ (11\% of total) mapped to unique sites in the genome ('hits', shown in Additional file 1). Despite our efforts to minimize resequencing WGA primers, 30\% of non-mapping reads contained at least 11 bases of either Illumina primer or the proprietary Sigma WGA sequence. The remaining non-mapping reads could be either contaminated by shorter stretches of primer or reflected misassembled or missing regions of the genome. Of the uniquely-mapped reads, nearly $70 \%$ mapped to scaffolds previously localized to the $\mathrm{p}$ arm of chromosome 7 by the genetic map [10], with the majority of those scaffolds showing high $\mathrm{read} / \mathrm{kb}$ values (see Additional file 2). Since the published genetic map contains large gaps on chromosome 7, chromosome 2 and elsewhere, we also collated available cytogenetic map data from Fluorescence In Situ Hybridization with Tyramide Signal Amplification (FISHTSA) analysis using cDNA probes $[8,10,20]$, and evaluated read density on scaffolds anchored by known physical location of probes. Read density was congruent with the locations of all 90 physically-mapped scaffolds, including 4 markers in the $7 \mathrm{p}$ region (Figure 2, Additional file 3). Near the centromere (genetically mapped to $69 \mathrm{cM}+/-1 \mathrm{cM}$ [12]), this ratio drops from approximately 70 to 17 uniquely-mapped reads/kb, probably due to increased repeat density diluting unique sequence in centromeric scaffolds. We conservatively used the hit ratio of 17 unique reads/kb observed for Scaffold_298, localized by FISH to just under the centromere [10], as the actual border giving the lowest acceptable hit/kb threshold. All scaffolds with lower than $17 \mathrm{hits} / \mathrm{kb}$ were considered false positives (2164 scaffolds containing $6.3 \%$ of hits, Table 1 ). The area between 72 and $73 \mathrm{cM}$ contains scaffolds with around $1 \mathrm{read} / \mathrm{kb}$ indicating the laser cutting path. Below this borderline the hit/kb ratio rapidly falls more than $100 \times$.

$3.47 \%$ of unique hits $(135,487)$ were found in three scaffolds localized by the meiotic linkage map to non-7p regions. Two of these scaffolds were placed on the $q$ arm of chromosome 7 in the linkage map despite relatively high $\mathrm{read} / \mathrm{kb}$ values in our analysis (scaffold_598, 74.32 cM), 64 hits/kb and scaffold_1153, $96 \mathrm{cM}, 57$ hits/kb). The third scaffold (scaffold_302) mapped to the q arm of chromosome 8 (38.54 cM, 59 reads $/ \mathrm{kb}$ ). Since the high $\mathrm{hit} / \mathrm{kb}$ ratios of these scaffolds are similar to those previously mapped to $7 \mathrm{p}$ region by FISH-TSA (scaffold_827, 75 reads/kb; scaffold_266, 31 reads/kb; scaffold_271, 79 reads/kb; and scaffold_75, 50 reads/kb) [10], we hypothesize that these sequences should be reassigned to the laser microdissected $7 \mathrm{p}$ arm. In total, we identified 231 v4.1 scaffolds not represented on the genetic map (22.5\% of unique hits) with a read/kb value higher than 17 (border scaffold_298) which we can assign to the 7p region. 29 of these unmapped scaffolds are larger than 100 kb. On the other hand, 13 scaffolds with markers on $7 \mathrm{p}$ in the genetic map bore lower $\mathrm{read} / \mathrm{kb}$ values than the threshold defined above, more consistent with a nonchromosome $7 \mathrm{p}$ location.

\section{Identification of hybrid scaffolds}

To identify misassembled (hybrid or broken) scaffolds, uniquely mapped reads were visualized on the $X$. tropicalis 4.1 assembly (Additional file 4). If a whole scaffold were present in the microdissected chromosome part, its full length should be covered by reads. Hybrid scaffolds may show gaps without any chromosome-specific hits, consistent with these sequences deriving from other chromosomal regions. We found that approximately $15 \%$ of scaffolds with meiotic map markers on $7 p$ contained gaps in read coverage larger than $100 \mathrm{~kb}$. These gaps in unique read hits are not generally caused by increased repeat density, which is similar in hit-rich and hit-absent areas (Figure 3), and the gaps usually contain genes, also suggesting that they are not due to repetitive sequence. FISH-TSA analysis of three suspected hybrid scaffolds (75, 266 and 270) confirmed assembly discrepancies, with probes from 'hit rich' regions localized to $7 \mathrm{p}$, but cDNA probes from 'hit absent' areas decorating chromosomes 3 and 4 (Figure 3). Meiotic map markers from these 


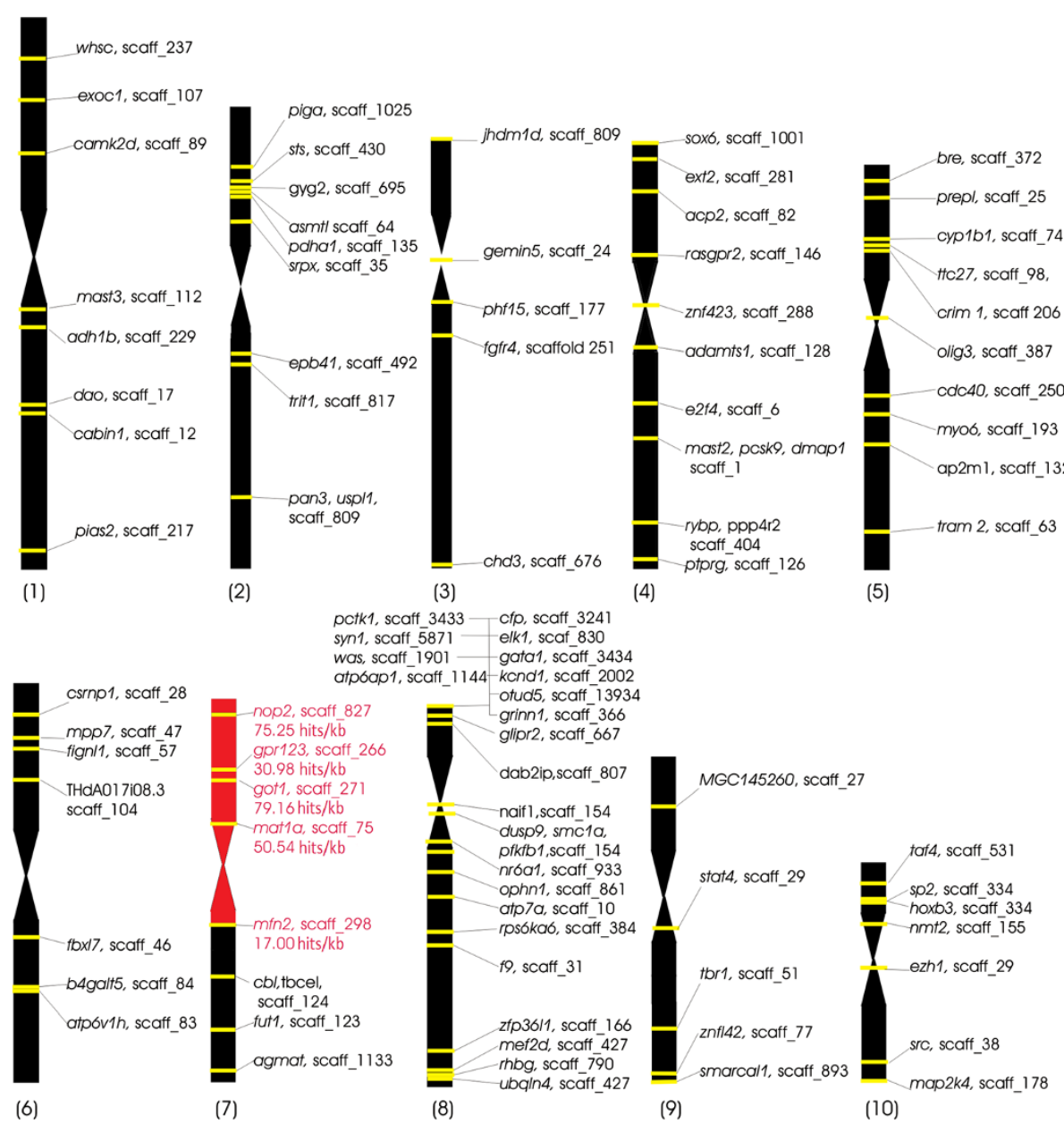

Figure 2 Uniquely-localized read distribution on physically mapped scaffolds. Schematic of $X$. tropicalis karyotype showing genes mapped by FISH-TSA [8,10,20] and cognate v4.1 scaffolds (scaff_number). High read density (17-80 hits/kb) is seen for scaffolds localized to the microdissected region (red region). Genes that physically localized to non-7p regions were all contained by scaffolds with $<1$ hit/kb (black regions).

scaffolds are also present in $7 p$ and non- $7 p$ regions, confirming that these are hybrid 'broken' scaffolds rather than mapping errors. Our analysis thus helps pinpoint bad sequence joins in the scaffold assembly.

\section{Comparison to v7.1 assembly}

The v7.1 assembly incorporates both sequence information, long-range contiguity from the meiotic map, and gene synteny relationships from amniote genomes. 75\% of the coverage has been ordered provisionally into 10 large superscaffolds corresponding to the $10 \mathrm{X}$. tropicalis chromosomes. In our analysis, 4,489,728 reads placed uniquely on the v7.1 assembly (see Additional file 5). $80.8 \%$ of uniquely-mapping reads localized to scaffold (chromosome) 7, with $91 \%$ of these in the $0-60 \mathrm{Mb}$ area roughly corresponding to the $7 \mathrm{p}$ region (Figure 4 ) with an average hit/kb ratio of 29.23 . We defined this value (29 reads/kb) as a threshold for hit-positive v7.1 scaffolds. Five large gaps with much lower read density were observed between 0-0.7 Mb, 3.2-4.5 Mb, 5.2-6.7 Mb, 19.7-21.3 $\mathrm{Mb}$ and 49.7-55.2 Mb, consistent with areas that were misassembled in v7.1. The $60-65 \mathrm{Mb}$ area is the approximate location of the presumptive laser path, but misassembly in this region is also possible. In the

Table 1 Reads locating uniquely to Xenopus tropicalis meiotically-mapped v4.1 scaffolds

\begin{tabular}{lcccc}
\hline All hits (\%) & $\begin{array}{c}\text { Hits to 7p v4.1 } \\
\text { scaffolds (\%) }\end{array}$ & $\begin{array}{c}\text { Hits to above threshold } \\
\text { non-7p v4.1 scaffolds (\%) }\end{array}$ & $\begin{array}{c}\text { Hits to above threshold } \\
\text { unmapped scaffolds (\%) }\end{array}$ & $\begin{array}{c}\text { Hits to under-threshold } \\
\text { scaffolds (\%) }\end{array}$ \\
\hline $3,900,340(100 \%)$ & $2,638,303$ & $135,487(3.47 \%)$ & 880,547 & 24,6003 \\
& $(67.64 \%)$ & & $(22.58 \%)$ & $(6.3 \%)$ \\
\hline
\end{tabular}

Threshold is set to17 hit/kb (hit/kb of bordering scaffold_298). 


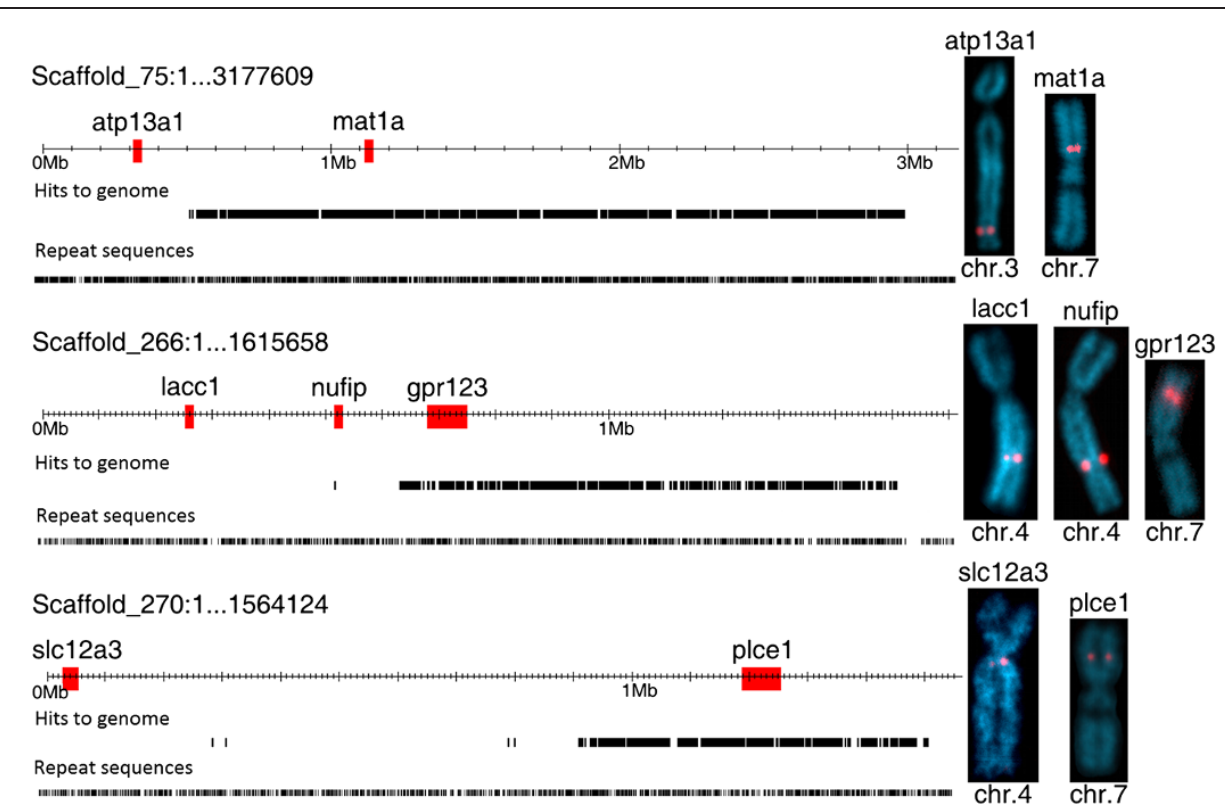

Figure 3 FISH-TSA analysis of three hybrid scaffolds. Selected genes from read-rich and read-absent areas of v4.1 scaffolds_75, _266, and _270 were physically mapped to $X$. tropicalis chromosomes using specific cDNA probes. Top line shows probe gene location on scaffold, second line shows distribution of uniquely-mapping reads, third line shows distribution of repetitive sequence (similar in hit-rich and hit-absent areas).

remaining part of scaffold 7 corresponding to $7 \mathrm{q}(65-$ $120 \mathrm{Mb})$, we identified four large read-dense regions (67.7-68.8 Mb, 32 hits/kb; 76.5-77.0 Mb, 54 hits/kb; 79.3-81.0 Mb, 17 hits/kb; and 106.0-107.0 Mb, 57 hits/ $\mathrm{kb})$ which are likely to be located on the other arm of chromosome 7. Similar sharply-demarcated candidate 7p areas with high read/kb ratios were found also in chromosome/scaffolds 1 (114.0-115 Mb, 45 hits/kb), 3 (36.7-38.0 Mb, 62. hits/kb, see Figure 4) and 4. (11.0$11.5 \mathrm{Mb}, 39 \mathrm{hits} / \mathrm{kb}, 14.5-15.35 \mathrm{Mb}, 34$. hits/kb) (shown in Additional file 6). Analysis of 'orphan' scaffolds not incorporated into the main chromosomal assembly identified another 14 orphan scaffolds larger than $100 \mathrm{~kb}$ showing high hit/kb values comparable to scaffold 7 . Of

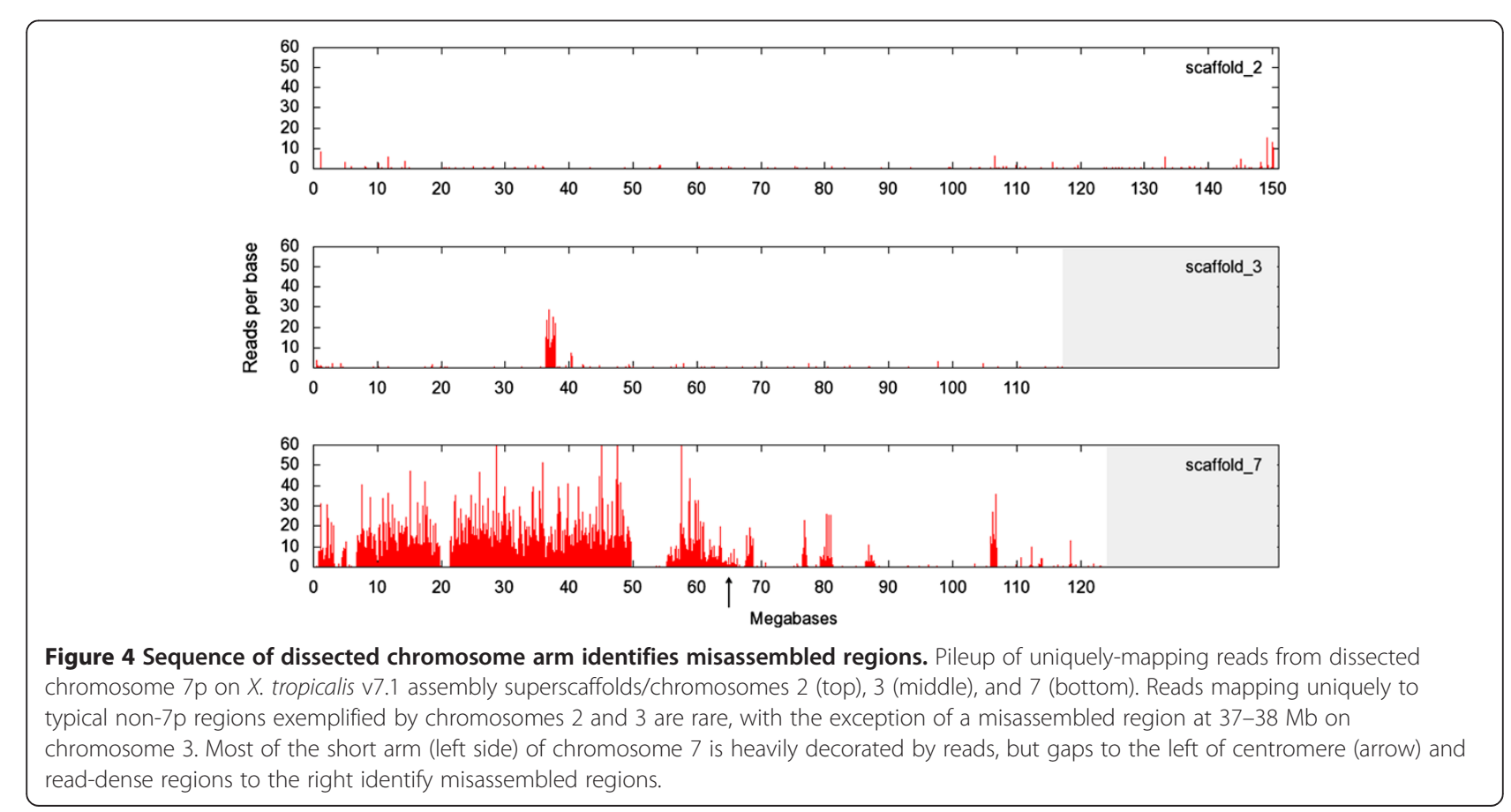


these, scaffold 35 (27 hit/kb) is covered by high-density reads only on $30 \%$ of its length, consistent with a bad sequence join.

\section{Discussion}

We present an improved technique for next generation sequencing of laser microdissected chromosome arms. Comparing a previous study [18] using 454 sequencing of DOP-PCR amplified human chromosome arms which yielded similar 80 bp average reads, we obtained approximately $30,000 \times$ more reads and $10,000 \times$ more unique hits to genome. The increase in usable reads allowed us to set high stringency conditions for mapping (97.5\% identity).

As mentioned above, a large portion of the reads that did not map to the genome contained WGA primer sequences, despite precautions to minimize sequencing end fragments by the use of the Nextera system for building the sequencing library. Further optimization of library construction to avoid ends could increase usable read yield considerably.

The yield of mapped reads was comparable to that of a study where wheat flow-sorted chromosome arms were sequenced [21]. The flow sorting approach can collect abundant chromosomal DNA, but can only distinguish a minority of chromosomes in a given karyotype based on size alone [16]. Laser microdissection enables visual control and much greater discrimination among similarly-sized chromosomes, for example sorting by $\mathrm{p} / \mathrm{q}$ arm ratio in metaphase spreads or using banding techniques. We verified our analysis using available FISH data for 93 relevant v4.1 scaffolds $[8,10,20]$. All known $7 \mathrm{p}$ scaffolds have a high unique $\mathrm{read} / \mathrm{kb}$ ratio, whereas non-7p scaffolds show a maximum of $1 \mathrm{hit} / \mathrm{kb}$ without exceptions (see Figure 2 and Additional file 3). The total fraction of above-threshold reads mapping to chromosome $7 \mathrm{p}$ was $93.7 \%$ in the $\mathrm{v} 4.1$ assembly and $91 \%$ in v7.1, comparable to an analysis of human chromosome 19 [22] where 93\% of reads mapped to the cognate region.

As mentioned above, the chromosome 7 linkage map contains a large gap between the most distal marker $\left(\begin{array}{ll}0 & \mathrm{cM}\end{array}\right)$ and the next one at $15 \mathrm{cM}$. We identified 264 scaffolds from the 4.1 assembly in $7 \mathrm{p}$, only 49 of which are represented in the current linkage map. The remaining 215 scaffolds, with a total of size $17 \mathrm{Mb}$, had either unknown or incorrect positions. Many of these newly localized scaffolds are likely to be contained in the distal gap as well as smaller gaps elsewhere on the $7 p$ linkage map.

Although $\mathrm{v} 4.17 \mathrm{p}$ scaffolds show unique $\mathrm{read} / \mathrm{kb}$ ratios ranging from 17 to 527, these values are sufficient to assign scaffolds to the laser microdissected area. Identification of scaffolds as hybrid or misassembled by virtue of unequal hit coverage was verified by FISH-TSA. Scaffolds close to the laser cut have a lower coverage (17-20 hits/kb), but these hits are evenly distributed across the scaffold. Misassembled scaffolds in the central part of $7 p$ (unaffected by the laser) show sharply uneven distribution of reads, with some areas showing values of $\sim 30-40$ hit/kb and others $<1 \mathrm{hit} / \mathrm{kb}$.

In our analysis of the v7.1 assembly, 91\% of uniquelymapping reads were to above-threshold ( 29 hits/kb) scaffolds, a lower percentage than when compared with the v4.1 assembly and map (see Table 2). Our analysis suggests that this is largely due to hits in a few defined regions that were misassembled in v7.1 chromosomescale superscaffolds 1, 3 and 4, leading to overall belowthreshold values for those entire chromosomes/scaffolds. Our analysis also identifies 97 above-threshold orphan scaffolds, contributing an additional 7.2 $\mathrm{Mb}$ of sequence to the $7 \mathrm{p}$ region.

Analysis of sequence from microdissected chromosome arms identified errors in both available $X$. tropicalis assemblies, with at least $15 \%$ of $\mathrm{v} 4.1$ scaffolds mapped to $7 \mathrm{p}$ scaffolds misassembled. In the v7.1 assembly, we located large regions of $7 p$ sequence which were misassigned to superscaffolds 1, 3, and 4 (Additional file 6). Interestingly, v4.1 scaffolds_75,_266, and_270, identified as hybrids by our analysis, were divided in the v7.1 assembly. However, FISH analysis using probes corresponding to the atp13a1 and lacc1 genes from hit-absent regions of v4.1 scaffolds_75 and _266 revealed actual locations on chromosomes 3 and 4, respectively, rather than the positions given by the v7.1 assembly on chromosomes/superscaffolds 8 and 2. These results suggest that the v7.1 assembly has successfully identified bad sequence joins, but has not necessarily correctly repaired them in all cases. High throughput sequencing of microdissected chromosomes or chromosomal arms helps to identify such misassembled domains, as well as to assign orphan scaffolds to chromosomal regions. Microdissection and sequencing of particularly problematic areas, such as $7 p$ and $2 p$, allows sequence domains to be assigned to a specific chromosome arm

Table 2 Reads locating uniquely to Xenopus tropicalis v7.1 assembly

\begin{tabular}{lccc}
\hline All hits (\%) & Hits to v7.1 superscaffold 7 (\%) & $\begin{array}{c}\text { Hits to above-threshold orphan } \\
\text { scaffolds (\%) }\end{array}$ & $\begin{array}{c}\text { Hits to under-threshold } \\
\text { scaffolds (\%) }\end{array}$ \\
\hline $4,489,728$ & $3,627,889$ & 461,438 & 228,745 \\
$(100 \%)$ & $(80.8 \%)$ & $(10.26 \%)$ & $(8.94 \%)$ \\
\hline
\end{tabular}

Threshold set to $29 \mathrm{hit} / \mathrm{kb}$ (hit/kb of scaffold 7). 
before extrapolating position from meiotic map or synteny data. Improving assembly of the short arm of chromosome 7 is critical for characterizing the sexdetermination genes of Xenopus tropicalis, which is known to use a different system from the $D M-W$ mechanism found in $X$. laevis [23].

Since our method could be combined with chromosome banding, it is likely to be particularly useful for de novo assembly of challenging genome projects, such as that of the allotetraploid laboratory model Xenopus laevis $(N=18)$. In the absence of meiotic or physical map information, correct regional assembly and longrange contiguity would be enhanced by dissecting and sequencing specific chromosomes, all of which in $X$. laevis can be unambiguously distinguished by banding pattern [24].

\section{Conclusions}

We have demonstrated feasibility of high-throughput sequencing from as little as 15 microdissected chromosome arms. This approach will be helpful for validating and completing problematic regions in the $X$. tropicalis genome, and can also be used in other species without sequenced genomes for describing gene content in selected chromosomes or providing long-range contiguity. Moreover, the technique is applicable to molecular analysis of isolated chromosomes from small numbers of cells, which is important for investigation of haplotypes or molecular rearrangements in clinical cytogenetics or oncology.

\section{Methods}

\section{Chromosomal spreads for laser microdissection}

$X$. tropicalis chromosome nomenclature followed [20]. Metaphase spreads were prepared from euploid primary cell cultures of dissected testes as described in [20] with minor changes. Cells were trypsinized and hypotonized in $38 \mathrm{mM} \mathrm{KCl}$ for $5 \mathrm{~min}$. After fixation, cell suspensions were stored overnight at $-20^{\circ} \mathrm{C}$. For laser microdissection, cells were dropped on a polyethylene naphthalene membrane (P.A.L.M. GmbH, Bernried, Germany) attached to a thin glass slide, allowed to dry, and stained with $3 \%$ Giemsa in $\mathrm{H}_{2} \mathrm{O}$ for 10 min.

\section{Laser microdissection}

Chromosomes were harvested as in [25]. Briefly, 15 copies of the $\mathrm{p}$ arm of chromosome 7 were microdissected and collected using a PALM MicroLaser system (P.A.L.M. $\mathrm{GmbH}$, Bernried, Germany) coupled with an inverted microscope (Olympus) under an oil immersion objective (100× magnification). Chromosome arms were catapulted by a single laser pulse directly into the cap of a PCR tube containing $4 \mu \mathrm{L}$ PCR oil. To ensure that the whole short arm of chromosome 7 was obtained, the laser cut was targeted to the $\mathrm{q}$ arm border of the centromere region.

\section{Whole genome amplification}

An initial round of whole genome amplification was performed using the WGA4 GenomePlex Single Cell Kit (Sigma-Aldrich). Dissected chromosome arms were digested with Proteinase K, followed by library preparation and amplification according to manufacturer's instructions. Primary PCR products were cleaned up using a Qiaquick Gel Extraction Kit (QIAGEN) column. 20 ng of primary WGA4 product was then reamplified using the WGA3 system (GenomePlex WGA Reamplification Kit, Sigma-Aldrich) according to our original protocol for preparation of $X$. tropicalis painting probes [26]. The secondary PCR product was purified by ethanol precipitation, yielding approximately $20 \mu \mathrm{g}$ of DNA fragments averaging 500-600 bp in size.

\section{High throughput sequencing and library construction}

In order to minimize resequencing WGA adaptors at ends of amplified fragments, libraries for high-throughput sequencing were constructed by in vitro transposition to simultaneously fragment the DNA and introduce sequencing primer/adaptors using the Nextera DNA sample prep kit (Illumina, Inc.) according to manufacturer's instructions. $50 \mathrm{ng}$ (measured by QuBit, Life Technology) of dissected chromosome amplification product DNA was used with Nextera Illumina-Compatible Enzyme Mix and low molecular weight buffer to generate libraries with fragment size of $\sim 200$ to 400 bp (including the 135 bp adapter sequence), and enriched by limited-cycle PCR. Library quality was determined by QuBit, Agilent Bioanalyser and QT-PCR using KAPA library quantification kit (KAPABiosystems, Boston, USA) before loading $6.5 \mathrm{pM}$ on a lane of a GAII flow cell for sequencing $80 \mathrm{bp}$ single reads.

\section{Data analysis}

Reads were mapped to Xenopus tropicalis v4.1 and v7.1 assemblies (available on www.xenbase.org) [27] using Bowtie (http://bowtie-bio.sourceforge.net) [28,29]. Due to prevalence of repeats in the $X$.tropicalis genome, only unique hits with higher than $97.5 \%$ identity were selected, using Bowtie parameters $\mathrm{m}=1$ and $\mathrm{v}=2$. The sequences obtained were counted and analyzed using PERL scripts and Microsoft Excel. Repetitive genomic regions were obtained from the UCSC (browser http://genome.ucsc.edu/).

FISH-TSA (Fluorescence In Situ Hybridization with Tyramide Signal Amplification).

Metaphase spreads for FISH-TSA analysis were prepared from the same euploid testes cell cultures as for 
the laser microdissection procedure, using the FISHTSA protocol described in [30].

\section{Additional files}

Additional file 1: Reads mapping uniquely to v4.1 assembly. Table showing all unique hits to 4.1 scaffolds, ordered by hit/kb ratio.

Additional file 2: Reads localizing uniquely to meiotically-mapped chromosome 7 scaffolds. Chromosome 7 meiotic map with polymorphic markers and associated Xenopus tropicalis v4.1 scaffolds are shown with hits/kb from dissected chromosome $7 p$ sequence. Read density decreases sharply near $72 \mathrm{cM}$, just beneath centromere at $69 \mathrm{cM}+/-1 \mathrm{cM}$.

Additional file 3: Uniquely-mapped read distribution on physical map. All genes previously mapped by FISH-TSA $[8,10,20]$ are shown with associated chromosome/linkage group (chrom/LG), meiotic map position, cognate v4.1 scaffolds with hit/kb ratio, probe size (Ampl. Length), relative distance from centromere (RDC), and v7.1 position. All regions with high read density (17-80 hits/kb, marked in red) are located on $7 p$. FISH-TSA probes in all other regions (black) have $<1$ hit/kb.

Additional file 4: Reads mapping uniquely to v4.1 scaffolds. The file includes all v4.1 scaffolds larger than $100 \mathrm{~kb}$ with hit/kb>17. Every panel contains one scaffold with its length scale, position of all genes, and visualization of reads.

Additional file 5: Reads mapping uniquely to v7.1 assembly. Table showing all unique hits to v7.1 scaffolds. Chromosome-scale superscaffolds are shown top right, with orphan scaffolds on left ordered by decreasing unique reads $/ \mathrm{kb}$ ratio.

Additional file 6: Read distribution on v7.1 scaffolds. The file includes all scaffolds larger than $100 \mathrm{~kb}$ with unique reads $/ \mathrm{kb}>29.23$, including scaffold 35 and regions with high coverage in superscaffolds 1 , 2, 3, 4 and 8. Scaffolds are ordered by ascending scaffold number. Superscaffold 7 is divided into several smaller parts. Each scaffold shows length scale and reads.

\section{Competing interests}

The authors declare that they have no competing interests.

\section{Authors' contributions}

$V K, E S$ and LZ designed the project; ES, SK, HC, AS, and TT performed the experiments; ES, NO, VZ \& MG analyzed the data; ES, VK and LZ wrote the paper. All authors read and approved the final manuscript.

\section{Acknowledgement}

We are grateful to Joachim Kurth (NIMR Division of Developmental Biology) for technical assistance. The research was funded by grants from the Grant Agency of Charles University in Prague 407311; Grant Agency of Czech Republic P502/11/P522; Ministry of Education, Youth and Sports of Czech Republic MSM0021620858; UNCE 204013; SW 265211; Ministry of Agriculture of the Czech Republic MZE 0002716202 and UK Medical Research Council U117560482 and U117597137.

\section{Author details}

${ }^{1}$ Charles University in Prague, Faculty of Science, Vinicna 7, Prague 2128 44, Czech Republic. ${ }^{2}$ MRC National Institute for Medical Research, The Ridgeway, Mill Hill, London NW7 1AA, UK. ${ }^{3}$ Veterinary Research Institute, Hudcova 70, Brno 621 00, Czech Republic

Received: 12 February 2013 Accepted: 24 May 2013

Published: 28 May 2013

\section{References}

1. Pagani I, Liolios K, Jansson J, Chen IM, Smirnova T, Nosrat B, Markowitz VM, Kyrpides NC: The Genomes OnLine Database (GOLD) v. 4: status of genomic and metagenomic projects and their associated metadata. Nucleic Acids Res 2012, 40:D571-D579.
2. Barthelson R, McFarlin AJ, Rounsley SD, Young S: Plantagora: modeling whole genome sequencing and assembly of plant genomes. PLoS One 2011, 6:e28436.

3. Harland RM, Grainger RM: Xenopus research: metamorphosed by genetics and genomics. Trends Genet 2011, 27:507-515.

4. Kashiwagi K, Kashiwagi A, Kurabayashi A, Hanada H, Nakajima K, Okada M, Takase M, Yaoita Y: Xenopus tropicalis: an ideal experimental animal in amphibia. Exp Anim 2010, 59:395-405.

5. Amaya E: Xenomics. Genome Res 2005, 15:1683-1691.

6. Carruthers S, Stemple DL: Genetic and genomic prospects for Xenopus tropicalis research. Semin Cell Dev Biol 2006, 17:146-153.

7. Abu-Daya A, Khokha MK, Zimmerman LB: The hitchhiker's guide to Xenopus genetics. Genesis 2012, 50:164-175.

8. Mácha J, Teichmanová R, Sater AK, Wells DE, Tlapáková T, Zimmerman LB, Krylov V: Deep ancestry of mammalian $X$ chromosome revealed by comparison with the basal tetrapod Xenopus tropicalis. BMC Genomics 2012, 13:315.

9. Hellsten U, Harland RM, Gilchrist MJ, Hendrix D, Jurka J, Kapitonov V, Ovcharenko I, Putnam NH, Shu S, Taher L, et al: The genome of the Western clawed frog Xenopus tropicalis. Science 2010, 328:633-636.

10. Wells DE, Gutierrez L, Xu Z, Krylov V, Macha J, Blankenburg KP, Hitchens M, Bellot L, Spivey M, Stemple DL, et al: A genetic map of Xenopus tropicalis. Dev Biol 2011, 354:1-8.

11. Gilchrist MJ: From expression cloning to gene modeling: the development of Xenopus gene sequence resources. Genesis 2012, 50:143-154.

12. Olmstead AW, Lindberg-Livingston A, Degitz SJ: Genotyping sex in the amphibian, Xenopus (Silurana) tropicalis, for endocrine disruptor bioassays. Aquat Toxicol 2010, 98:60-66.

13. Bewick AJ, Chain FJ, Zimmerman LB, Sesay A, Gilchrist MJ, Owens ND, Seifertova E, Krylov V, Macha J, Tlapakova T, et al: A large pseudoautosomal region on the sex chromosomes of the frog Silurana tropicalis. Genome Biol Evol 2013. Epub ahead of print.

14. Li R, Fan W, Tian G, Zhu H, He L, Cai J, Huang Q, Cai Q, Li B, Bai Y, et al: The sequence and de novo assembly of the giant panda genome. Nature 2010, 463:311-317.

15. Dalloul RA, Long JA, Zimin AV, Aslam L, Beal K, Blomberg LA, Bouffard P, Burt DW, Crasta O, Crooijmans RP, et al: Multi-platform next-generation sequencing of the domestic turkey (Meleagris gallopavo): genome assembly and analysis. PLOS Biol 2010, 8:e1000475.

16. Doležel J, Vrána J, Safár J, Bartoš J, Kubaláková M, Simková H: Chromosomes in the flow to simplify genome analysis. Funct Integr Genomics 2012, 12:397-416.

17. Ma L, Xiao Y, Huang H, Wang Q, Rao W, Feng Y, Zhang K, Song Q: Direct determination of molecular haplotypes by chromosome microdissection. Nat Methods 2010, 7:299-301.

18. Weise A, Timmermann B, Grabherr M, Werber M, Heyn P, Kosyakova N, Liehr T, Neitzel H, Konrat K, Bommer C, et al: High-throughput sequencing of microdissected chromosomal regions. Eur J Hum Genet 2010, 18:457-462.

19. Höckner M, Erdel M, Spreiz A, Utermann G, Kotzot D: Whole genome amplification from microdissected chromosomes. Cytogenet Genome Res 2009, 125:98-102.

20. Khokha MK, Krylov V, Reilly MJ, Gall JG, Bhattacharya D, Cheung CY, Kaufman S, Lam DK, Macha J, Ngo C, et al: Rapid gynogenetic mapping of Xenopus tropicalis mutations to chromosomes. Dev Dyn 2009, 238:1398-1346.

21. Hernandez P, Martis M, Dorado G, Pfeifer M, Gálvez S, Schaaf S, Jouve N, Šimková H, Valárik M, Doležel J, Mayer KF: Next-generation sequencing and syntenic integration of flow-sorted arms of wheat chromosome $4 \mathrm{~A}$ exposes the chromosome structure and gene content. Plant J 2012, 69:377-386.

22. Yang $\mathrm{H}$, Chen $\mathrm{X}$, Wong WH: Completely phased genome sequencing through chromosome sorting. Proc Natl Acad Sci U S A 2011, 108:12-17.

23. Yoshimoto S, Okada E, Umemoto H, Tamura K, Uno Y, Nishida-Umehara C, Matsuda Y, Takamatsu N, Shiba T, Ito M: A W-linked DM-domain gene, DM-W, participates in primary ovary development in Xenopus laevis. Proc Natl Acad Sci U S A 2008, 105:2469-2474.

24. Schmid M, Steinlein C: Chromosome banding in Amphibia. XVI. Highresolution replication banding patterns in Xenopus laevis. Chromosoma 1991, 101:123-132.

25. Kubickova S, Cernohorska H, Musilova P, Rubes J: The use of laser microdissection for the preparation of chromosome-specific painting probes in farm animals. Chromosome Res 2002, 10:571-577. 
26. Krylov V, Kubickova S, Rubes J, Macha J, Tlapakova T, Seifertova E, Sebkova $\mathrm{N}$ : Preparation of Xenopus tropicalis whole chromosome painting probes using laser microdissection and reconstruction of $\mathrm{X}$. laevis tetraploid karyotype by Zoo-FISH. Chromosome Res 2010, 18:431-439.

27. Xenbase: Xenopus laevis and Xenopus tropicalis biology and genomics resource. [www.xenbase.org]

28. Langmead B, Trapnell C, Pop M, Salzberg SL: Ultrafast and memoryefficient alignment of short DNA sequences to the human genome. Genome Biol 2009, 10:R25.

29. Bowtie: an ultrafast, memory-efficient short read aligner. http://bowtie-bio. sourceforge.net/.

30. Krylov $\mathrm{V}$, Tlapakova T, Macha J: Localization of the single copy gene Mdh2 on Xenopus tropicalis chromosomes by FISH-TSA. Cytogenet Genome Res 2007, 116:110-112.

doi:10.1186/1471-2164-14-357

Cite this article as: Seifertova et al:: Efficient high-throughput

sequencing of a laser microdissected chromosome arm. BMC Genomics 2013 14:357.

\section{Submit your next manuscript to BioMed Central and take full advantage of:}

- Convenient online submission

- Thorough peer review

- No space constraints or color figure charges

- Immediate publication on acceptance

- Inclusion in PubMed, CAS, Scopus and Google Scholar

- Research which is freely available for redistribution 